\title{
Order-disorder oscillations in exciton-polariton superfluids
}

\author{
Hiroki Saito, Tomohiko Aioi, and Tsuyoshi Kadokura \\ Department of Engineering Science, University of Electro-Communications, Tokyo 182-8585, Japan
}

(Dated: August 19, 2018)

\begin{abstract}
The dynamics of an exciton-polariton superfluid resonantly pumped in a semiconductor microcavity are investigated by mean-field theory. Modulational instability develops into crystalline order and then ordered and disordered states alternately form. A supersolid-like state is also found, in which superflow coexists with crystalline order at rest.

PACS numbers: 71.36.+c, 47.54.-r, 64.60.Cn, 67.80.K-
\end{abstract}

\section{INTRODUCTION}

Transitions between ordered and disordered states can be controlled by changing the parameters of a system. For example, a liquid can be solidified and then melted back into a liquid by controlling the temperature or pressure. Another example is a flow pattern in the wake of an obstacle. A periodic vortex street can change into turbulence by increasing the Reynolds number and turbulence can transform back into a vortex street by decreasing the Reynolds number. In a large complex system, order and disorder can alternately appear in a partial system, such as clouds in the sky. However, in a simple laboratory system, such repeated transitions between ordered and disordered states usually do not occur unless an external parameter is changed $\stackrel{1}{*}$. Ordering phenomena or pattern formations occur in a wide variety of nonequilibrium dissipative systems ${ }^{2}$, such as Bénard convection cells ${ }^{3}$ and chemical patterns in reaction diffusion systems 4,5 . However, once a pattern has been generated in these systems, it is preserved if the parameters are fixed, although it may vary with time. By contrast, we here propose a system in which crystalline order and disorder alternately emerge despite external parameters being fixed.

The system that we consider is an exciton-polariton superfluid in a planar semiconductor microcavity ${ }^{6.7}$. There have been many recent experimental studies of this system, including: bistable properties ${ }^{8,9}$, BoseEinstein condensation ${ }^{10,11}$, Bogoliubov excitations ${ }^{12}$, quantized vortices ${ }^{13,14}$, superfluidity ${ }^{15}$, quantum hydrodynamics $^{16,17}$, and pattern formation ${ }^{18,19}$. Of the various ways to create a polariton superfluid, we consider a system of polaritons pumped by a quasi-resonant continuous-wave laser. Since polaritons have a short lifetime of $\sim 10 \mathrm{ps}$, which is comparable to the time scale of the dynamics, the system is a nonequilibrium dissipative system with continuous feeding. It is thus suitable for studying nonlinear phenomena far from equilibrium.

In this paper, we investigate pattern formation and destruction dynamics in an exciton-polariton superfluid. The polaritons are first pumped to the lower branch of the bistability ${ }^{8,9}$, which is an unstable stationary state ${ }^{20}$. Modulational instability then arises and a hexagonal crystalline pattern develops, which eventually collapses, destroying the order. However, the pattern reappears from the disordered distribution and grows again. Subsequently, the ordered and disordered distributions emerge alternately. We also show that the crystalline order can remain at rest even in the presence of superflow.

This paper is organized as follows. Section II formulates the problem, Section III demonstrates numerical results, and Section IV gives conclusions of this study.

\section{FORMULATION OF THE PROBLEM}

Excitons in quantum wells and photons confined between Bragg mirrors are assumed to be described by macroscopic wave functions $\psi_{\mathrm{X}}$ and $\psi_{\mathrm{C}}$, obeying the twodimensional nonlinear Schrödinger equations given by ${ }^{20}$

$$
\begin{aligned}
i \hbar \frac{\partial \psi_{\mathrm{X}}}{\partial t}= & \hat{H}_{0}^{\mathrm{X}} \psi_{\mathrm{X}}+\hbar \Omega_{\mathrm{R}} \psi_{\mathrm{C}}+g\left|\psi_{\mathrm{X}}\right|^{2} \psi_{\mathrm{X}}-i \hbar \frac{\gamma_{\mathrm{X}}}{2} \psi_{\mathrm{X}} \\
i \hbar \frac{\partial \psi_{\mathrm{C}}}{\partial t}= & \hat{H}_{0}^{\mathrm{C}} \psi_{\mathrm{C}}+\hbar \Omega_{\mathrm{R}} \psi_{\mathrm{X}}-i \hbar \frac{\gamma_{\mathrm{C}}}{2} \psi_{\mathrm{C}} \\
& +\hbar F(\boldsymbol{r}) e^{i\left(\boldsymbol{k}_{\mathrm{p}} \cdot \boldsymbol{r}-\omega_{\mathrm{p}} t\right)}
\end{aligned}
$$

where $\hat{H}_{0}^{j}$ and $\gamma_{j}$ are respectively the free Hamiltonians and the decay rates of an exciton $(j=\mathrm{X})$ and a photon $(j=\mathrm{C}), \Omega_{\mathrm{R}}$ is the Rabi frequency, and $g$ is the exciton-exciton interaction coefficient. The free Hamiltonians are approximated as $\hat{H}_{0}^{\mathrm{X}}=\hbar \omega_{0}^{\mathrm{X}}$ and $\hat{H}_{0}^{\mathrm{C}}=$ $\hbar \omega_{0}^{\mathrm{C}}-\hbar^{2} \nabla^{2} /\left(2 m_{\mathrm{C}}\right)$, where $m_{\mathrm{C}}$ is the in-plane effective mass of a cavity photon. Diagonalizing the first and second terms on the right-hand side (rhs) of Eq. (1), we obtain the dispersion relations $\omega_{ \pm}(k)$ for the upper and lower free polaritons. The last term on the rhs of Eq. (1a) describes pumping by an external laser beam with a profile $F(\boldsymbol{r})$, in-plane wave vector $\boldsymbol{k}_{\mathrm{p}}$, and frequency $\omega_{\mathrm{p}}$.

We numerically solve Eq. (1) using the pseudospectral $\operatorname{method}^{21}$. As an initial state, small white noise is set to $\psi_{\mathrm{X}}$ and $\psi_{\mathrm{C}}$ to exclude exact numerical symmetry. In the following calculations, we only consider the case of normal incidence of the pump beam on the sample, i.e., $\boldsymbol{k}_{\mathrm{p}}=0$. The detuning of the pump frequency from the lower polariton is defined as $\delta=\omega_{\mathrm{p}}-\omega_{-}(0)$. We use the 

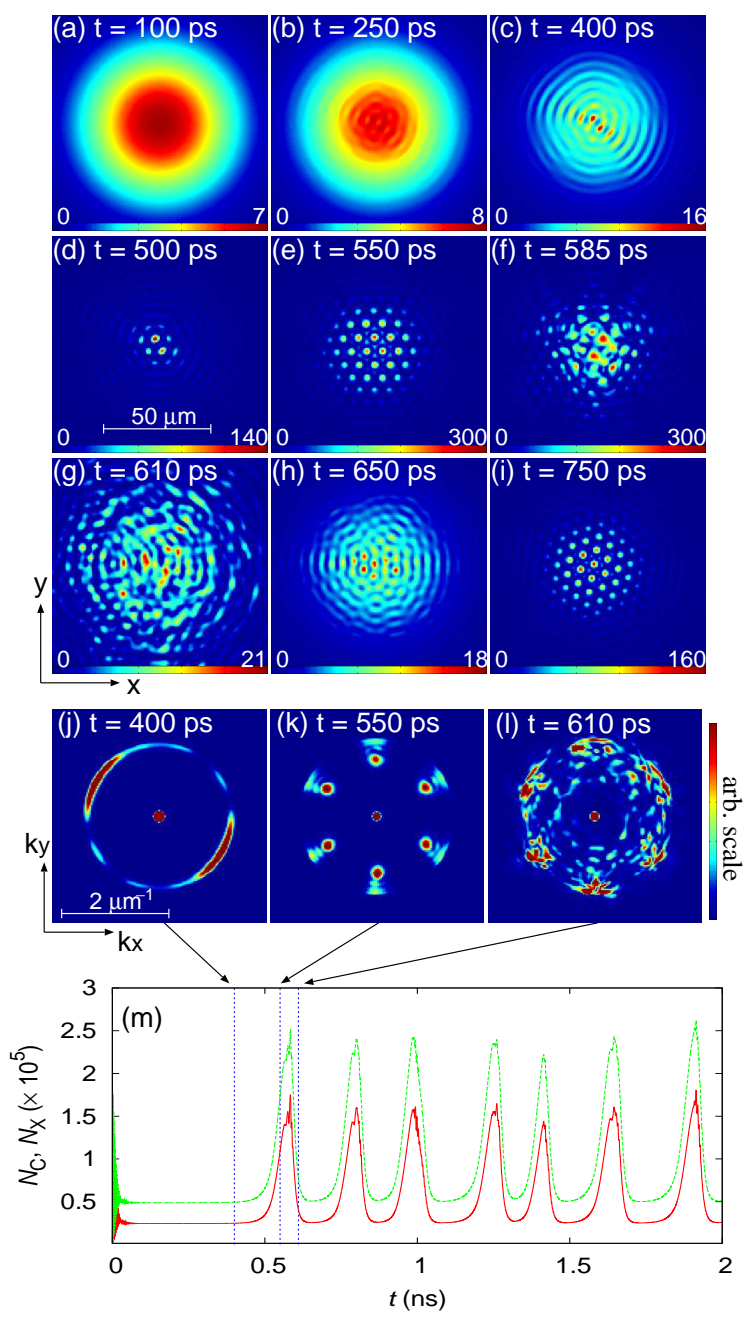

FIG. 1: (color online) (a)-(i) Snapshots of the density profile $\left|\psi_{\mathrm{C}}\right|^{2}$ of photons. The pump parameters in Eq. (2) are $\hbar F_{0}=$ $8.97 \mathrm{meV}$ and $d=50 \mu \mathrm{m}$. The color scale in each image is normalized by the peak density, where the unit of density is $\mu \mathrm{m}^{-2}$. The field of view of each panel is $100 \times 100 \mu \mathrm{m}$. (j)-(l) Fourier-transformed profiles $\left|\int \psi_{\mathrm{C}} e^{-i \boldsymbol{k} \cdot \boldsymbol{r}} d \boldsymbol{r}\right|^{2}$, where the field of view is $4 \times 4 \mu \mathrm{m}^{-1}$. (m) Time evolution of $N_{\mathrm{C}}=\int\left|\psi_{\mathrm{C}}\right|^{2} d \boldsymbol{r}$ (red solid line) and $N_{\mathrm{X}}=\int\left|\psi_{\mathrm{X}}\right|^{2} d \boldsymbol{r}$ (green dashed line). The vertical dotted lines show $t=400,550$, and 610 ps. See supplemental material ${ }^{22}$.

Gaussian profile of the pump beam as

$$
F(\boldsymbol{r})=F_{0} \exp \left[-\left(x^{2}+y^{2}\right) / d^{2}\right],
$$

where $F_{0}$ is proportional to the peak intensity and $d$ is the $1 / e$ width. The parameters are $m_{\mathrm{C}}=2 \times 10^{-5} m_{\mathrm{e}}$ with $m_{\mathrm{e}}$ being the electron mass, $\hbar \Omega_{\mathrm{R}}=5 \mathrm{meV}, g=$ $0.01 \mathrm{meV}_{\mu \mathrm{m}^{2}}$, and $\hbar \delta=1.53 \mathrm{meV}$. For simplicity, we assume $\omega_{0}^{\mathrm{X}}=\omega_{0}^{C}$ and $\gamma \equiv \gamma_{\mathrm{X}}=\gamma_{\mathrm{C}}=(10 \mathrm{ps})^{-1}$.

\section{NUMERICAL RESULTS}

Figures 1(a)-1(i) show the dynamics of the density profile $\left|\psi_{\mathrm{C}}(\boldsymbol{r})\right|^{2}$ of photons $\left(\left|\psi_{\mathrm{X}}(\boldsymbol{r})\right|^{2}\right.$ is always similar to $\left|\psi_{\mathrm{C}}(\boldsymbol{r})\right|^{2}$; it will not be shown henceforth). First, the polariton density grows in the region of $r \lesssim d$ and reaches a steady state with a Gaussian profile (Fig. 11(a)), reflecting the pump profile in Eq. (2). Modulational instability then arises around the center of the pump region and a pattern develops (Figs. 1(b) and 1(c)). At this stage, the pattern has no crystalline symmetry. At $t \simeq 500$ ps, crystalline order emerges around the center and it grows outward to form a triangular lattice (Figs. 1(d) and 1(e)). The wave function has almost the same phase at each density peak. This ordered state is not a steady state and the density of each peak continues to increase. When the peak density reaches some critical value, the triangular lattice abruptly collapses, destroying the crystalline order (Figs. 1(f) and 1(g)). The disturbance decays with time and a similar modulational pattern to that in Fig. 1(c) re-emerges (Fig. 1(h)), which is followed by the reappearance of crystalline order (Fig. 1(i)). The collapse and restoration of crystalline order are subsequently repeated.

Figure 11(m) shows the time evolution of the norms $N_{\mathrm{C}}=\int\left|\psi_{\mathrm{C}}\right|^{2} d \boldsymbol{r}$ and $N_{\mathrm{X}}=\int\left|\psi_{\mathrm{X}}\right|^{2} d \boldsymbol{r}$. After the initial fluctuations for $t \lesssim 100 \mathrm{ps}, N_{\mathrm{C}}$ and $N_{\mathrm{X}}$ reach plateaus corresponding to the steady state in Fig. 1(a). The Fourier-transformed profile of this steady state localizes within $|k| \lesssim 0.1 \mu \mathrm{m}^{-1}$ (data not shown). As the modulational instability grows, a ring-shaped profile emerges at $|k| \simeq 1.3 \mu \mathrm{m}^{-1} \simeq 2 \pi /(5 \mu \mathrm{m})$ (Fig. 11 $\left.(\mathrm{j})\right)$, which reflects the pattern in Fig. 1(c). The anisotropic shape of the ring depends on the small noise added to the initial state. As the crystalline order grows, $N_{\mathrm{C}}$ and $N_{\mathrm{X}}$ increase rapidly and the ring in Fourier space changes to hexagonal peaks (Fig. 1 (k)). Since the peaks in Fig. 1 (k) are closer to the origin than the ring in Fig. 1(j), the period of the triangular lattice in Fig. 1(e) is larger than the modulation wavelength in Fig. 1(c). The sudden reduction in $N_{\mathrm{C}}$ and $N_{\mathrm{X}}$ corresponds to the collapse of the triangular lattice, where the six hexagonal peaks change to a disordered distribution in Fourier space (Fig. 1(1)). The disordered components are subsequently cleaned up and the ring shape grows, which is followed by hexagonal peaks.

To qualitatively understand the behaviors shown in Fig. 1] we consider the lower-polariton approximation. Neglecting the upper polariton branch, we can reduce Eq. (11) $\mathrm{to}^{20}$

$$
\begin{aligned}
i \hbar \frac{\partial \psi_{\mathrm{LP}}}{\partial t}= & \left(-\frac{\hbar^{2}}{2 m_{\mathrm{LP}}} \nabla^{2}+g_{\mathrm{LP}}\left|\psi_{\mathrm{LP}}\right|^{2}-i \hbar \frac{\gamma}{2}\right) \psi_{\mathrm{LP}} \\
& +\hbar F_{\mathrm{LP}} e^{-i \omega_{\mathrm{p}} t}
\end{aligned}
$$

where $\psi_{\mathrm{LP}}=X_{\mathrm{LP}} \psi_{\mathrm{X}}+C_{\mathrm{LP}} \psi_{\mathrm{C}}, m_{\mathrm{LP}}=2 m_{\mathrm{C}}, g_{\mathrm{LP}}=$ $\left|X_{\mathrm{LP}}\right|^{4} \mathrm{~g}$, and $F_{\mathrm{LP}}=C_{\mathrm{LP}} F$ are the macroscopic wave function, the effective mass, the interaction coefficient, 
and the pump intensity of the lower polaritons, respectively. $X_{\mathrm{LP}}$ and $C_{\mathrm{LP}}$ are the Hopfield coefficients. For simplicity, we consider the case of homogeneous pumping and the polariton wave function can be divided into

$$
\psi_{\mathrm{LP}}(\boldsymbol{r}, t)=e^{-i \omega_{\mathrm{p}} t}\left[\phi_{\mathrm{Ss}}+\delta \phi(\boldsymbol{r}, t)\right] .
$$

Substituting Eq. (41) into Eq. (3), we find that the homogeneous steady state $\phi_{\mathrm{ss}}$ is determined by

$$
\left(\hbar \delta-g_{\mathrm{LP}}\left|\phi_{\mathrm{ss}}\right|^{2}+i \hbar \gamma / 2\right) \phi_{\mathrm{ss}}=\hbar F_{\mathrm{LP}},
$$

which will have multiple solutions of $\phi_{\mathrm{ss}}$ for a certain range of $F_{\mathrm{LP}}$ if $\delta / \gamma>\sqrt{3} / 2$. For the parameters in Fig. 1. $\delta / \gamma \simeq 23$ and Eq. (5) has three solutions for 0.8 $\mathrm{meV} \lesssim \hbar F_{\mathrm{LP}} \lesssim 14 \mathrm{meV}$. The steady state in Fig. 1(a) corresponds to the state with the lowest density of the three solutions.

We use Bogoliubov analysis to investigate the stability of the steady state. The first order of $\delta \phi$ in Eq. (3) gives the Bogoliubov-de Gennes equation. For an excitation of the form $\delta \phi(\boldsymbol{r}, t)=u_{k} e^{-i \omega t+i \boldsymbol{k} \cdot \boldsymbol{r}}+v_{k}^{*} e^{i \omega^{*} t-i \boldsymbol{k} \cdot \boldsymbol{r}}$, the Bogoliubov-de Gennes equation can be diagonalized to yield the excitation frequency:

$$
\omega=-i \frac{\gamma}{2} \pm \sqrt{\left(\omega_{k}-\delta+\omega_{\mathrm{ss}}\right)\left(\omega_{k}-\delta+3 \omega_{\mathrm{ss}}\right)},
$$

where $\omega_{k}=\hbar k^{2} /\left(2 m_{\mathrm{LP}}\right)$ and $\omega_{\mathrm{ss}}=g_{\mathrm{LP}}\left|\phi_{\mathrm{ss}}\right|^{2} / \hbar$. When the imaginary part of $\omega$ is positive, the corresponding mode grows exponentially. From Eq. (6), the condition for this modulational instability to occur is found to be $\omega_{\mathrm{ss}}>\gamma / 2$ and $\delta-2 \omega_{\mathrm{ss}}+\left(\omega_{\mathrm{ss}}^{2}-\gamma^{2} / 4\right)^{1 / 2}>0$, which is satisfied when $r \lesssim 20 \mu \mathrm{m}$ in the situation shown in Fig. 1(a). For $\omega_{k}=\delta-2 \omega_{\mathrm{ss}}$, Eq. (6) has the largest imaginary part, which corresponds to the most unstable mode. For the parameters in Fig. 1(a), the most unstable wavelength is estimated to be $\simeq 7 \mu \mathrm{m}$, which is in reasonable agreement with the modulation in Fig. 1(b).

The hexagonal crystalline order shown in Figs. 1(e) and 1 (i) is a manifestation of the nonlinear effect. To see this, we simplify the problem using a wave function with the form

$$
\psi_{\mathrm{LP}}(\boldsymbol{r}, t)=e^{-i \omega_{\mathrm{p}} t} \phi_{\mathrm{SS}}[1+c(t) f(\boldsymbol{r})],
$$

where $f(\boldsymbol{r})=\sum_{i} \cos \boldsymbol{k}_{i} \cdot \boldsymbol{r}$. Substituting Eq. (7) into Eq. (3) and extracting terms proportional to $f(\boldsymbol{r})$, we obtain

$$
\begin{aligned}
i \dot{c}= & \left(\omega_{k}-\omega_{\mathrm{p}}-i \gamma / 2\right) c+\omega_{\mathrm{ss}}\left[2 c+c^{*}+A\left(2|c|^{2}+c^{2}\right)\right. \\
& \left.+B|c|^{2} c\right],
\end{aligned}
$$

where $A$ and $B$ are constants depending on $f(\boldsymbol{r})$. The $O(c)$ terms in Eq. (8), which give Eq. (6), are responsible for the exponential growth of $c$ and we restrict the wave number $\left|\boldsymbol{k}_{i}\right|$ to be the most unstable one. If $A \neq 0, O\left(c^{2}\right)$ terms will become important as $c$ grows. Equation (8) gives

$$
\frac{d}{d t}|c|^{2}=-\gamma|c|^{2}+i \omega_{\mathrm{ss}}\left(c^{2}-c^{* 2}\right)+i \omega_{\mathrm{ss}} A|c|^{2}\left(c-c^{*}\right),
$$
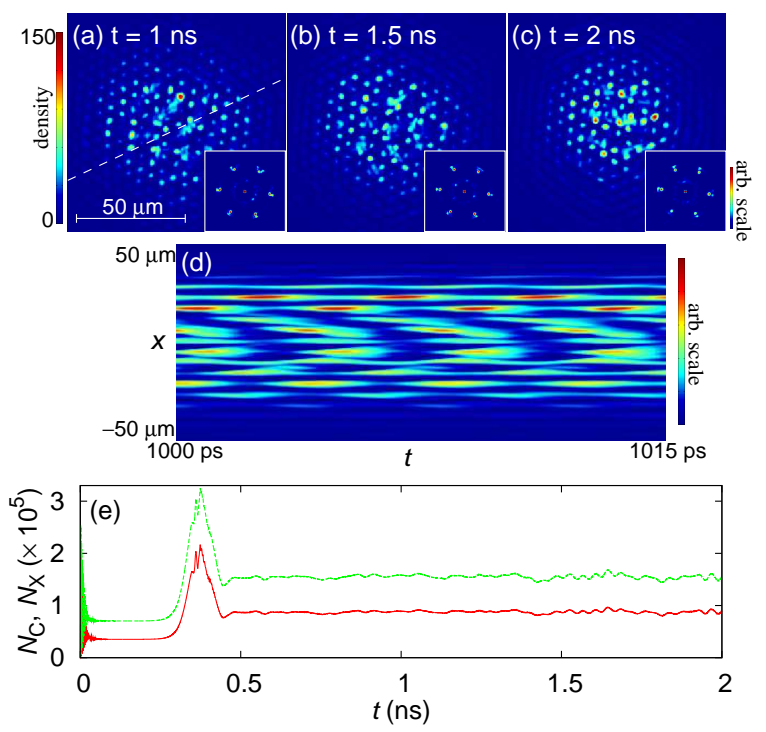

FIG. 2: (color online) (a)-(c) Snapshots of the density profile $\left|\psi_{\mathrm{C}}\right|^{2}$ for $d=60 \mu \mathrm{m}$. The insets show Fourier-transformed profiles. The field of view of the main panels is $100 \times 100 \mu \mathrm{m}$ and that of the insets is $4 \times 4 \mu \mathrm{m}^{-1}$. (d) Spatiotemporal image of the density on the dashed line in (a). (e) Time evolution of the norms $N_{\mathrm{C}}$ (red solid line) and $N_{\mathrm{X}}$ (green dashed line). In (a)-(e), all the parameters except $d$ are the same as those in Fig. 11 See supplemental material ${ }^{22}$.

which indicates that the solution satisfying $i A\left(c-c^{*}\right)>0$ dominates the exponential growth, breaking the symmetry with respect to $c \rightarrow-c$ in the linear part of Eq. (8). The simplest form of $f(\boldsymbol{r})$ with nonzero $A$ is $f(\boldsymbol{r})=\cos \boldsymbol{k}_{1} \cdot \boldsymbol{r}+\cos \boldsymbol{k}_{2} \cdot \boldsymbol{r}+\cos \boldsymbol{k}_{3} \cdot \boldsymbol{r}$ with $\boldsymbol{k}_{1}+\boldsymbol{k}_{2}+\boldsymbol{k}_{3}=0$; i.e., the three wave vectors form a regular triangle. In this case, $A=1$ and $B=15 / 4$. Thus, the hexagonal order dominates the growth as the modulation amplitude grows. The term of $B$ in Eq. (8) effectively shifts the resonance by $\omega_{\mathrm{ss}} B|c|^{2}$. This is why the peak wave number decreases (Fig. 1(k)) as the hexagonal pattern grows.

We return to the numerical simulations of Eq. (11). When the peak density grows and exceeds some critical density, the subsequent behaviors can be classified into three types. The first one is the behavior shown in Fig. 1, where the hexagonal order and disorder alternately emerge. The second behavior is entrainment into the large density for a large pump intensity $F_{0}$. When the local peak density reaches the uppermost branch of Eq. (5), the whole system is entrained into the uppermost branch and the dynamics settle down there. The third behavior occurs when the width $d$ of the pump beam is large, which is shown in Fig. 2, After the hexagonal pattern is formed and destroyed at $t \simeq 400 \mathrm{ps}$ in a manner similar to Figs. 1(a)-1(f), the collapse and restoration of the peaks occur locally and sporadically, which results in the pattern shown in Figs. 2(a)-2(c). This is in contrast with the dynamics in Fig. 1] where the order-disorder oscillation occurs synchronously in the whole system. Figure 2(d) shows a spatiotemporal image of the density 


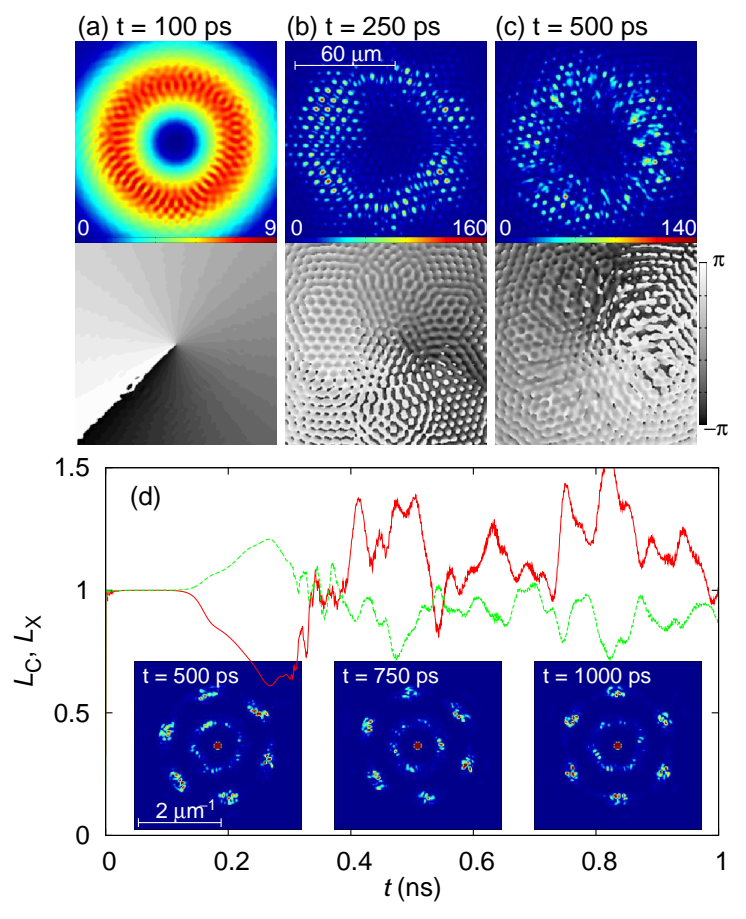

FIG. 3: (color online) (a)-(c) Snapshots of the density $\left|\psi_{\mathrm{C}}\right|^{2}$ and phase $\arg \psi_{\mathrm{C}}$ profiles for Laguerre-Gaussian pumping. The pump parameters in Eq. (10) are $\hbar F_{0}=21 \mathrm{meV}$ and $d=50 \mu \mathrm{m}$. The field of view of each panel is $120 \times 120$ $\mu \mathrm{m}$. (d) Time evolution of the normalized angular momenta $L_{j}=-i \int \psi_{j}^{*} \partial_{\theta} \psi_{j} d \boldsymbol{r} / \int\left|\psi_{j}\right|^{2} d \boldsymbol{r}$ of photons $(j=C$, red solid line) and excitons $(j=X$, green dashed line). The insets show Fourier-transformed profiles, where the field of view is $4 \times 4 \mu \mathrm{m}^{-1}$. Except for the pump function, the parameters are the same as those in Fig. 1 See supplemental material ${ }^{22}$.

$\left|\psi_{\mathrm{C}}\right|^{2}$ on the dashed line in Fig. 2(a). Each density peak blinks periodically with a period $\simeq 4$ ps. This may be related with the blinking phenomenon observed in experiments ${ }^{8}$. Since the peaks grow and collapse at various places, the norms $N_{\mathrm{C}}$ and $N_{\mathrm{X}}$ fluctuate around their averages, as shown in Fig. 2(e). There is also a case in which several cycles of the synchronous order-disorder oscillations shown in Fig. 1 occur, followed by the local oscillations shown in Fig. 2,

We next examine polariton pumping with an angular momentum using a Laguerre-Gaussian beam. The pump function is given by

$$
F(\boldsymbol{r})=F_{0} \frac{x+i y}{d} \exp \left[-\left(x^{2}+y^{2}\right) / d^{2}\right] .
$$

The pumped polaritons form a ring-shaped density profile with a phase $\theta=\arg (x+i y)$ and the modulational instability grows along the ring, as shown in Fig. 3(a). The triangular lattice initially has some mismatches along the ring, as shown in Fig 3(b), since the lattice grows from different points on the ring. Subsequently, in a man- ner similar to Fig. 2, the peaks collapse and revive locally (Fig. [3(c)). The angular momenta and the Fouriertransformed profiles shown in Fig. 3(d) exhibit an interesting behavior. Although the angular momentum per particle is $\sim \hbar$ on average, from which the angular velocity for a typical radius $R \simeq 40 \mu \mathrm{m}$ is estimated to be $\dot{\theta} \simeq \hbar /\left(m_{\mathrm{LP}} R^{2}\right) \simeq 1.8 \mathrm{rad} / \mathrm{ns}$, the hexagonal pattern in Fig. [3(d) does not rotate; rather, it drifts slightly in the opposite direction (clockwise). This indicates that the hexagonal crystalline order is almost irrotational despite the rotation of the superflow 23 ; this may be regarded as a supersolid-like behavior.

In experiments, a disorder potential in a sample typically has an energy of at least $\sim 0.1 \mathrm{meV}$ and a wavelength of $\sim 10 \mu \mathrm{m}^{24}$. We have numerically confirmed that dynamics similar to Figs. 1 3 are observed even when the disorder potential is added to the rhs of Eq. (1a). Under continuous pumping of polaritons such as in the present situation, it is experimentally difficult to perform time-resolved measurements. Time-integrated imaging will reduce the hexagonal order, since its direction is variable (see Figs. 1(e) and 1(i)) because of the rotational symmetry of the Hamiltonian. However, in reality, the disorder potential breaks the rotational symmetry and fixes the direction of the hexagonal order, which enables us to use time-integrated imaging to identify the hexagonal order. The order-disorder oscillation shown in Fig. 1 may also be detected by taking spatial and temporal correlations 17 .

\section{CONCLUSIONS}

We have investigated the dynamics of an excitonpolariton superfluid coherently pumped in a semiconductor microcavity. Under appropriate pumping, a hexagonal crystalline order is formed, destroyed, and restored; this cycle is repeated many times (Fig. 11). Such a spontaneous oscillation between ordered and disordered states is a unique phenomenon in a simple nonequilibrium open system. We have also shown that superflow can coexist with crystalline order at rest (Fig. 3), which is reminiscent of supersolidity. The continuously pumped excitonpolariton system thus affords a quantum dissipative system that is promising for studying novel dissipative structures.

\section{Acknowledgments}

This work was supported by Grants-in-Aid for Scientific Research (No. 22340116 and No. 23540464) from the Ministry of Education, Culture, Sports, Science and Technology of Japan. 
1 A mathematical model that exhibits order-disorder oscillation is proposed in J. H. Collet, Physica A 386, 345 (2007).

2 M. C. Cross and P. C. Hohenberg, Rev. Mod. Phys. 65, 851 (1993).

3 H. Bénard, Ann. Chim. Phys. 23 (Ser. 7), 62 (1900).

4 A. N. Zaikin and A. M. Zhabotinsky, Nature (London) 225, 535 (1970).

${ }^{5}$ Q. Ouyang and H. L. Swinney, Nature (London) 352, 610 (1991).

6 H. Deng, H. Haug, and Y. Yamamoto, Rev. Mod. Phys. 82, 1489 (2010).

7 I. Carusotto and C. Ciuti, arXiv:1205.6500.

8 A. Baas, J. Ph. Karr, H. Eleuch, and E. Giacobino, Phys. Rev. A 69, 023809 (2004).

9 N. A. Gippius, S. G. Tikhodeev, V. D. Kulakovskii, D. N. Krizhanovskii, and A. I. Tartakovskii, Europhys. Lett. 67, 997 (2004).

10 J. Kasprzak, M. Richard, S. Kundermann, A. Baas, P. Jeambrun, J. M. J. Keeling, F. M. Marchetti, M. H. Szymańska, R. André, J. L. Staehli, V. Savona, P. B. Littlewood, B. Deveaud, and Le Si Dang, Nature (London) 443, 409 (2006).

11 R. Balili, V. Hartwell, D. Snoke, L. Pfeiffer, and K. West, Science 316, 1007 (2007).

12 S. Utsunomiya, L. Tian, G. Roumpos, C. W. Lai, N. Kumada, T. Fujisawa, M. Kuwata-Gonokami, A. Löffler, S. Höfling, A. Forchel, and Y. Yamamoto, Nature Phys. 4, 700 (2008).

13 K. G. Lagoudakis, M. Wouters, M. Richard, A. Baas, I. Carusotto, R. André, Le Si Dang, and B. DeveaudPlédran, Nature Phys. 4, 706 (2008).

14 D. Sanvitto, F. M. Marchetti, M. H. Szymańska, G. Tosi,
M. Baudisch, F. P. Laussy, D. N. Krizhanovskii, M. S. Skolnick, L. Marrucci, A. Lemaître, J. Bloch, C. Tejedor, and L. Viña, Nature Phys. 6, 527 (2010).

15 A. Amo, J. Lefrére, S. Pigeon, C. Adrados, C. Ciuti, I. Carusotto, R. Houdré, E. Giacobino, and A. Bramati, Nature Phys. 5, 805 (2009).

16 G. Nardin, G. Grosso, Y. Léger, B. Piętka, F. MorierGenoud, and B. Deveaud-Plédran, Nature Phys. 7, 635 (2011).

17 A. Amo, S. Pigeon, D. Sanvitto, V. G. Sala, R. Hivet, I. Carusotto, F. Pisanello, G. Leménager, R. Houdré, E. Giacobino, C. Ciuti, and A. Bramati, Science 332, 1167 (2011).

18 D. Sanvitto, D. N. Krizhanovskii, D. M. Whittaker, S. Ceccarelli, M. S. Skolnick, and J. S. Roberts, Phys. Rev. B 73, 241308(R) (2006).

19 F. Manni, K. G. Lagoudakis, T. C. H. Liew, R. André, and B. Deveaud-Plédran, Phys. Rev. Lett. 107, 106401 (2011).

20 I. Carusotto and C. Ciuti, Phys. Rev. Lett. 93, 166401 (2004).

21 W. H. Press, S. A. Teukolsky, W. T. Vetterling, B. P. Flannery, Numerical Recipes, 3rd ed, Sec. 20.7 (Cambridge Univ. Press, Cambridge, 2007).

22 See supplemental material for the movies showing the dynamics of the density and Fourier transformed profiles, which are provided in the mpeg format.

23 A similar phenomenon is predicted in H. Saito, Y. Kawaguchi, and M. Ueda, Phys. Rev. Lett. 102, 230403 (2009).

24 G. Roumpos, M. D. Fraser, A. Löffler, S. Höfling, A. Forchel, and Y. Yamamoto, Nature Phys. 7, 129 (2011). 